\title{
INFLUENCIA DE LOS CAMBIOS CLIMÁTICOS EN LA DEFINICIÓN DEL SEXO EN EL AEDES AEGYPTI Y SU IMPACTO EN LAS EPIDEMIAS DE DENGUE Influence of climate changes on the definition of the sex of Aedes Aegypti and its impact on dengue epidemics
}

\author{
Ángel Ricardo Arenas Villamizar ${ }^{1}$, Luis Alexander Carvajal Pinilla ${ }^{2}$
}

Recibido: 27 de septiembre de 2012 - Aceptado: 24 de diciembre de 2012

${ }^{1}$ MSc Epidemiología Clínica, Universidad de la Frontera, Chile.

Médico y cirujano, Universidad

Surcolombiana. Jefe de UBN, Urgencias Clínica Universitaria,

Rafael Uribe Uribe. Cali - Colombia.

${ }^{2}$ Biólogo. MSc. Universidad Javeriana. Entomólogo. Investigador principal. Grupo Recursos Naturales. Corporación Universitaria del Huila. CORHUILA.

Correspondencia: angelrarster@gmail.com, ararenas@ccvul.org, lucarvaj@gmail.com

\section{Resumen}

Según la OMS el dengue es considerado como la más importante de las enfermedades virales transmitidas por mosquitos en el mundo. En los últimos 50 años, la incidencia se ha incrementado 30 veces. Según el informe de cambio climático y biodiversidad se estima que la temperatura media de la superficie terrestre ascienda entre un 1,4 y un $5,8^{\circ} \mathrm{C}$ para finales del siglo XXI. El objetivo del estudio fue establecer la influencia de la temperatura media ambiental en la determinación del sexo del Aedes aegypti y su influencia en las epidemias de dengue al influir en la determinación del sexo, como se ha evidenciado en algunas especies de lagartos y tortugas. Se realizó un estudio ecológico correlacional y un estudio experimental. En el primero se calculó un modelo de regresión lineal, entre la temperatura media ambiental de la ciudad de Neiva Colombia, entre los años 2008 y 2010 y la incidencia de dengue en los mismos períodos. En el análisis de resultados, el modelo de regresión calculado para el año 2009 el 33,10\% de la incidencia de dengue fue explicada por la temperatura media ambiental (Prob $>F=0.0007)$, para el año 2010, el modelo de regresión explicó el 26,05\% de la incidencia de dengue por la temperatura media ambiental (Prob $>F=0.0059)$. Según el modelo, el aumento de $1^{\circ} \mathrm{C}$ de la temperatura media ambiental explicó el aumentó en 11,78 casos de dengue. En el estudio experimental, se expusieron 600 huevos de Aedes aegypti a $26^{\circ} \mathrm{C}, 28^{\circ} \mathrm{C}$, y $32^{\circ} \mathrm{C}$ y se determinó el sexo hasta la segunda generación. Se calculó el Odds Ratio entre los resultados obtenidos a diferentes temperaturas $26^{\circ} \mathrm{C}, 28^{\circ} \mathrm{C}$ y $32^{\circ} \mathrm{C}$, encontrando que la oportunidad de ser hembra Vs. macho a $26^{\circ} \mathrm{C}$, fue de 0,78 veces ( $I C=0.52940-1.16612$ ), entre tanto que a $28^{\circ} \mathrm{C}$ fue de 1,78 veces (es decir $78 \%$ ) (IC $=1.242382 .55775)$. Puede existir una relación entre la temperatura media ambiental promedio de $28^{\circ} \mathrm{C}$ y el aumento en la incidencia de dengue por un aumento en la población de hembras de Aedes aegypti.

Palabras clave: dengue, Aedes aegypti, climatic change, sex. 


\begin{abstract}
According to the WHO, dengue is considered as the most important viral diseases transmitted by mosquitoes in the world. In the last 50 years, incidence has increased 30 times. According to the report about climate change and biodiversity, it is estimated that the average temperature of the earth's surface will rise between 1.4 and $5.8^{\circ} \mathrm{C}$ by the end of the twenty-first century. The aim of the study was to establish the influence of the environmental average temperature on determining the sex of the Aedes aegypti, and its impact on dengue epidemics, as it has been demonstrated in some species of lizards and tortoises. A correlational ecological study and an experimental study were carried out. The first one calculated a linear regression model between the environmental average temperature of Neiva, Colombia, from 2008 to 2010 and the incidence of dengue in the same periods. In the analysis of results, the regression model calculated for year 2009, $33.10 \%$ of the effect of dengue was explained by the environmental average temperature $($ Prob $>F=0.0007)$. For the year 2010, the regression model explained $26,05 \%$ of the incidence of dengue because of the environmental average temperature (Prob $>F=$ 0.0059). According to the model, the increase of $1^{\circ} \mathrm{C}$ of the environmental average temperature explained the increase in 11.78 cases of dengue. In the experimental study, 600 eggs of Aedes aegypti were exposed to $26^{\circ} \mathrm{C}, 28^{\circ} \mathrm{C}$ and $32^{\circ} \mathrm{C}$ and sex was determined incluidng the second generation. It was calculated the Odds Ratio between the gotten results at the above-mentioned exposure temperatures. I was found that the chance of being female vs. male at $26^{\circ} \mathrm{C}$ was 0.78 times $(C I=0.52940-1.16612)$, while it was 1.78 times (i.e. $78 \%)(I C=1.242382 .55775)$ at $28^{\circ} \mathrm{C}$. It is possible a relationship between the environmental average temperature at $28^{\circ} \mathrm{C}$ and the increase in the incidence of dengue because of a rise in the male population of Aedes aegypti.
\end{abstract}

Keywords: dengue, Aedes aegypti, climatic change, sex.

\section{INTRODUCCIÓN}

A nivel mundial, existe una preocupación creciente por los cambios climáticos relacionados con el llamado efecto invernadero, por lo que se estima que la temperatura media de la superficie terrestre ascienda entre un 1,4 y un $5,8^{\circ} \mathrm{C}$ para finales del siglo $\mathrm{XXI}^{(1)}$. Estos cambios ineludiblemente alterarán los ciclos de vida de muchas especies, y de aquellas como del mosquito Aedes aegypti, considerado el principal vector de enfermedades tropicales tales como dengue y fiebre amarilla. En estudios experimentales se ha encontrado que la Temperatura favorable para el desarrollo del Aedes aegypti se encuentra entre los 21 y 29 grados centígrados y la longevidad y fecundidad entre los 22 y los 30 grados centígrados $^{(2)}$. Por lo que se sugiere que la variación de la temperatura puede ser un factor estimulante de la eclosión y determinante en el favorecimiento del ciclo del Aedes aegypti ${ }^{(3-9)}$. Existe evidencia en cuanto a la relación de los cambios climáticos como el fenómeno del Pacífico (Fenómeno del Niño) en el comportamiento de las epidemias de dengue en el mundo(10-18). Los cambios 
climáticos y sus consecuencias sobre estos artrópodos jugarán un importante papel en la incidencia de dichas enfermedades en áreas donde en la actualidad no son endémicas. Para el tercer trimestre del año 2009 y hasta el tercer trimestre del año 2010, el dengue tuvo un comportamiento hiperendémico a nivel Nacional causando un impacto importante en la salud de la población, colapso de los recursos hospitalarios y altos costos de atención por aumento de la demanda de los mismos, evidenciando que las medidas de control existentes en la actualidad no son efectivas y mucho menos predictivas en la contención de las epidemias ${ }^{(19)}$. Se formuló la hipótesis que ciertas condiciones climáticas podrían relacionarse estrechamente con aumentos en la incidencia de la enfermedad por dengue al influir en la determinación del sexo en las etapas embrionarias tal como sucede en algunas especies de lagartos y tortugas ${ }^{(20-23)}$, por lo que al aumentar la temperatura por el efecto invernadero podrían aumentarse la relación hembras machos y por consiguiente aumentar las tasas de transmisión del virus. Estas variables climáticas pueden ser predictivas de epidemias al asociarse a condiciones climáticas específicas por períodos epidémicos de dengue, lo que podría optimizar el uso de los recursos disponibles en la actualidad.

\section{MATERIAles Y MÉTODOS}

La incidencia de dengue en el Departamento del Huila tuvo su mayor pico epidemiológico en el tercer trimestre del año 2009 hasta el tercer trimestre del año 2010. Teniendo en cuenta la evidencia que plantea una relación entre los cambios climáticos y las epidemias de dengue se planteó un estudio en dos fases.

Primera fase: Estudio descriptivo retrospectivo, ecológico correlacional en esta fase se calculó un modelo de regresión lineal para establecer si el aumento de los casos de dengue por semana epidemiológica era explicado por el aumento de la temperatura en la ciudad de Neiva, Huila Colombia entre los años 2008 y 2010.

Se tomaron las bases de datos del Instituto de Meteorología Hidrología y Estudios ambientales IDEAM de temperatura media ambiental y humedad relativa de la estación Benito Salas de la ciudad de Neiva registrados entre el 1 de Enero de 2008 y el 31 de Diciembre de 2010, tomando como datos la media de la temperatura ambiental en 24 horas por día y fueron agrupados por semanas epidemiológicas para establecer las condiciones climáticas en la misma semana de incidencia de dengue y hasta dos semanas antes (Figura 1) Los datos de la prevalencia de dengue se obtuvieron del

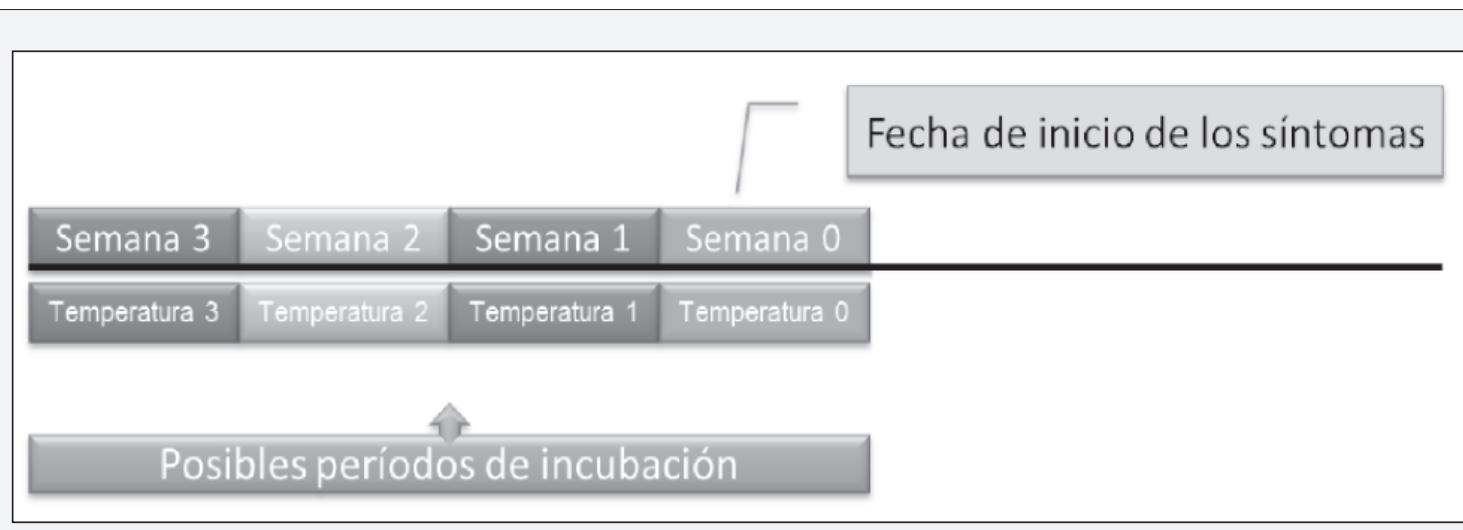

Figura 1. Modelo gráfico de condiciones climáticas del vector 3 semanas previas a la semana epidemiológica de dengue. 
Software SIVIGILA de la Secretaría de Salud Departamental del Huila, de pacientes con diagnóstico de dengue y dengue grave entre el año 2008 al año 2010, de la ciudad de Neiva tomando la prevalencia de casos por semanas epidemiológicas.

Con estas bases de datos se calculó un modelo de regresión lineal teniendo en cuenta que la variable respuesta (casos de dengue) es cuantitativa por lo tanto el modelo más adecuado para explicar el comportamiento de la variabilidad del fenómeno es la regresión lineal.

Los datos fueron procesados en el Software estadístico STATA Versión 10.0 para calcular el modelo de regresión lineal planteado para la prevalencia de dengue y la temperatura media ambiental por semanas epidemiológicas.

Segunda fase: Se realizó un estudio experimental en el cual cohortes de 600 huevos de cepas Rockefeller, de Aedes aegypti donados por el Instituto Nacional de Salud de Colombia fueron expuestos a condiciones controladas de temperatura y humedad $26^{\circ} \mathrm{C}, 28^{\circ} \mathrm{C}$ y $32^{\circ} \mathrm{C}$, para evaluar la influencia de la temperatura ambiental en condiciones experimentales en la determinación del sexo. Para ello se construyó una cámara de control ambiental, que permite la fluctuación de la temperatura interna en $0,5^{\circ} \mathrm{C}$, controlado por un termostato de gran precisión y éste conectado a un relevo que transmite la orden de encendido o apagado a una resistencia que genera la temperatura. Dicha resistencia se encuentra en un tubo de asbesto y se conecta a un ventilador que permite la oscilación constante del aire caliente al interior de la cámara. La humedad relativa externa, se controló con un aire acondicionado de 3.000 BTU, lo que evitó oscilaciones externas influenciadas por el medio ambiente. La temperatura se mantuvo en fotofase de 12 horas. Se expusieron las cepas a cada condición climática hasta la segunda generación F2, luego de lo cual fueron congelados los ejemplares y codificados, de acuerdo a los rangos de temperatura. La alimentación en fase de larva y pupa se realizó con concentrado canino 100 gramos; durante la etapa de mosquito se alimentaron con solución de glucosa y con sangre de ratas de laboratorio siguiendo el protocolo del Instituto Nacional de Salud de Colombia ${ }^{(24)}$ y teniendo en cuenta las consideraciones éticas para el uso de animales de laboratorio(25-27). Luego de obtener la segunda generación, los ejemplares fueron congelados y la determinación del sexo de los mismos la realizaron técnicos de Zoonosis de la Secretaría de Salud a quienes se les realizó previamente un estudio de concordancia para reducir la variabilidad de sus mediciones obteniendo una concordancia del $80 \%$. Posteriormente se realizó un doble enmascaramiento con la codificación de los ejemplares de los cuales los observadores no conocían su origen, ni tampoco los objetivos del estudio. Las divergencias de las observaciones fueron resueltas por un tercer observador experto entomólogo. A los resultados se calculó el odds ratio para determinar la oportunidad de ser hembra en las diferentes condiciones ambientales experimentales. El protocolo fue aprobado por el Comité de ética de la Universidad Surcolombiana de Neiva Huila, Colombia. Los modelos de regresión en la primera fase fueron calculados para cada prevalencia de dengue por semana con la temperatura media ambiental de hasta tres semanas antes comparando sus resultados. En la fase experimental el odds ratio fue calculado para cada rango de temperatura de exposición y comparados entre sí a $26^{\circ} \mathrm{C}, 28^{\circ} \mathrm{C}$ y $32^{\circ} \mathrm{C}$.

\section{RESUlTADOS}

De acuerdo con los datos de la Secretaría de Salud Departamental del Huila, la incidencia del dengue para el año 2008, no presentó períodos epidémicos.

Al calcular el modelo de regresión propuesto para este análisis se encuentra que no existe una significancia estadística para estos. El 


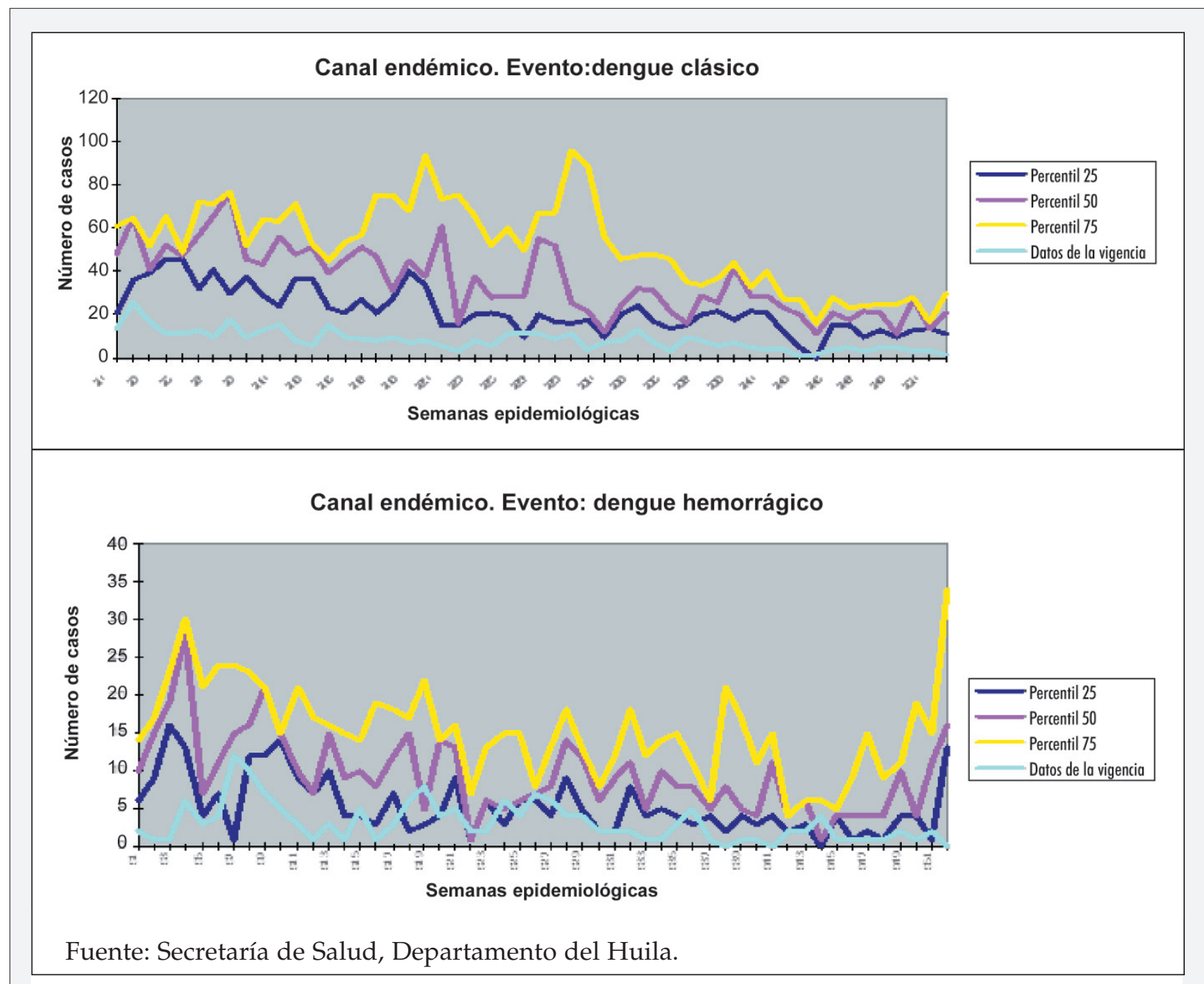

Figura 2. Canal endémico para dengue. Departamento del Huila. Año 2008.

modelo de regresión calculado para el año 2008 no presentó una significancia estadística $\mathrm{F}>0,05$ por lo que el modelo no explicó la incidencia de dengue por el aumento de la temperatura, para este período Rsquared $=-0.0561$.

En el año 2009 se presentó un período endémico a partir de la semana epidemiológica 30.

El modelo de regresión para el año 2009 presenta unos valores de $\mathrm{F}<0,05$ lo que lo hace estadísticamente significativo, explicando el $33,10 \%$ el comportamiento de la incidencia de dengue por las temperaturas, entre las temperaturas de 2 semanas previas a la presentación de los casos, según el modelo, el aumento de un grado centígrado $\left(1^{\circ} \mathrm{C}\right)$ de temperatura explicó el aumentó 3,5 casos de dengue con una $p$ de 0,040 .

Para las temperaturas de la semana 0 , el aumento de $1^{\circ} \mathrm{C}$ de temperatura explicó el aumentó 2,49 casos de dengue para esa semana epidemiológica

Para el año 2010 el período epidémico en el Departamento del Huila se extendió hasta la semana 43.

Para el año 2010 el coeficiente de correlación calculó una correlación entre la incidencia de dengue y la temperatura en la semana 0 del $48,51 \%$, del 39,90\% para la semana 1 del $27,18 \%$ para la semana 2 y del $22,21 \%$ para la semana 3. 
Tabla 1. Coeficiente de correlación de incidencia de dengue Vs. temperatura media ambiental Neiva Huila. Año 2008.

\begin{tabular}{cccccc}
\hline & casos & Temperatura 3 & Temperatura 2 & Temperatura 1 & Temperatura 0 \\
\hline casos & 10000 & & & \\
Temperatura 3 & $-0,0233$ & 1,0000 & & \\
p & 0,8736 & & & \\
Temperatura 2 & $-0,0845$ & 0,4119 & 1,0000 & 10000 \\
p & 0,5640 & 0,0033 & & \\
Temperatura 1 & 0,0878 & 0,1136 & 0,4112 & 10000 \\
p & 0,5484 & 0,4369 & 0,0033 & 0,4099 \\
Temperatura 0 & 0,1188 & 0,2330 & 0,1105 & 0,0034 \\
\hline
\end{tabular}


Tabla 2. Coeficiente de correlación de incidencia de dengue temperatura media ambiental. Neiva Huila. Año 2009.

\begin{tabular}{lccccc}
\hline & casos & Temperatura 0 & Temperatura 1 & Temperatura 2 & Temperatura 3 \\
\hline casos & 1.0000 & & & & \\
Temperatura 0 & 0,4921 & 1,0000 & & & \\
& 0,0003 & & & & \\
Temperatura 1 & 0,4388 & 0,6990 & 1,0000 & 1,0000 & 1,0000 \\
Temperatura 2 & 0,0014 & 0,0000 & & 0,7215 & \\
Temperatura 3 & 0,5066 & 0,6065 & 0,0000 & 0,0000 & \\
& 0,0002 & 0,0000 & 0,6178 & & \\
\hline
\end{tabular}

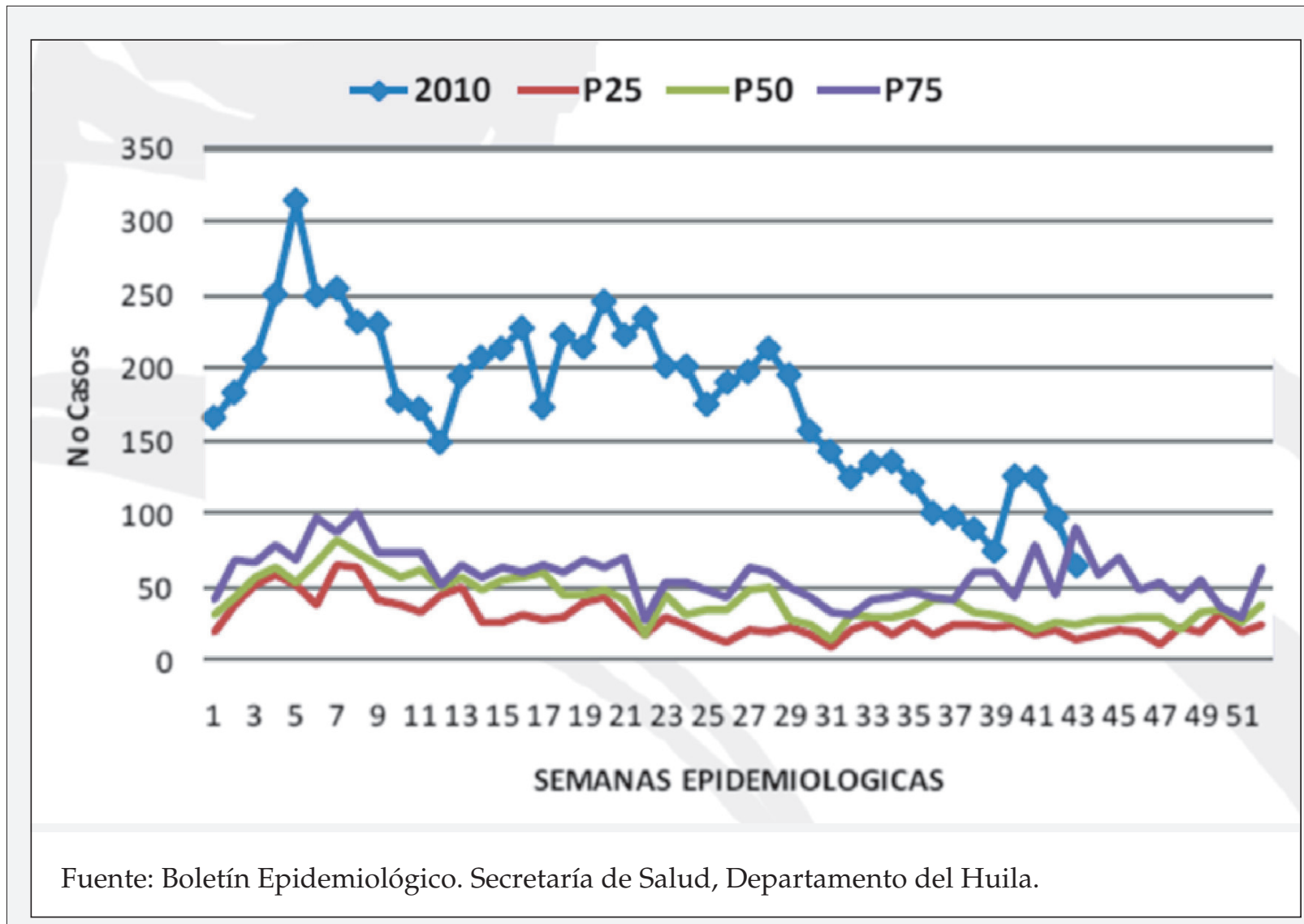

Figura 4. Canal endémico dengue. Departamento del Huila Año 2010. 
Tabla 3. Modelo de regresión incidencia de dengue temperatura media ambiental. Neiva Huila. Año 2009.

\begin{tabular}{|c|c|c|c|c|c|c|c|c|}
\hline \multirow[t]{2}{*}{ Source 1} & \multicolumn{2}{|c|}{ SS } & \multirow{3}{*}{$\begin{array}{c}\text { df } \\
4\end{array}$} & \multirow[t]{2}{*}{ MS } & & \multirow{3}{*}{\multicolumn{2}{|c|}{$\begin{array}{l}\text { Number of obs }= \\
F(4,47)= \\
\text { Prob }>F\end{array}$}} & 52 \\
\hline & & & & & & & & 5,81 \\
\hline Model & \multicolumn{2}{|c|}{1856.08174} & & \multicolumn{2}{|l|}{464.020435} & & & 0,0007 \\
\hline Residual & 375 & 84134 & 47 & 79.8051349 & & \multicolumn{2}{|c|}{ R-squared } & 0,3310 \\
\hline & & & & & & \multicolumn{2}{|c|}{ Adj R-squared } & 0,2741 \\
\hline Total 1 & \multicolumn{2}{|c|}{5606.92308} & 51 & 109.939668 & & \multicolumn{2}{|c|}{ Root MSE } & $=8,9334$ \\
\hline casos 1 & \multicolumn{3}{|c|}{ Coef. } & Std. Err. & $\mathrm{t}$ & $\mathrm{P}>1 \mathrm{t} l$ & \multicolumn{2}{|c|}{ [95\% Conf. Interval] } \\
\hline Temperatura & 3 & \multicolumn{2}{|l|}{-.5096144} & 1.505776 & $-0,34$ & 0,737 & -3.538845 & 2.519617 \\
\hline Temperatura & 2 & \multicolumn{2}{|l|}{3.57185} & 1.694326 & 2,11 & 0,040 & .1633059 & 6.980393 \\
\hline Temperatura & 1 & \multicolumn{2}{|l|}{-.2738963} & 1.653495 & $-0,17$ & 0,869 & -3.6003 & 3.052507 \\
\hline Temperatura & 0 & \multicolumn{2}{|l|}{2.494685} & 1.467259 & $1 ., 70$ & 0,096 & -.4570591 & 5.44643 \\
\hline cons 1 & \multicolumn{3}{|c|}{-120.0977} & 33.30397 & $-3,61$ & 0,001 & -187.0966 & -53.09874 \\
\hline
\end{tabular}

Tabla 4. Coeficiente de correlación de incidencia de dengue temperatura media ambiental. Neiva Huila. Año 2010.

\begin{tabular}{|c|c|c|c|c|c|}
\hline & casos & Temperatura 3 & Temperatura 2 & Temperatura 1 & Temperatura 0 \\
\hline casos & 10000 & & & & \\
\hline \multirow[t]{2}{*}{ Temperatura 3} & 0,2221 & 10000 & & & \\
\hline & 0,1136 & & & & \\
\hline \multirow[t]{2}{*}{ Temperatura 2} & 0,2718 & 0,7393 & 10000 & & \\
\hline & 0,0513 & 0,0000 & & & \\
\hline \multirow[t]{2}{*}{ Temperatura 1} & 0,3990 & 0,6846 & 0,7674 & 10000 & \\
\hline & 0,0034 & 0,0000 & 0,0000 & & \\
\hline \multirow[t]{2}{*}{ Temperatura 0} & 0,4851 & 0,6225 & 0,6930 & 0,7683 & 10000 \\
\hline & 0,0003 & 0,0000 & 0,0000 & 0,0000 & \\
\hline
\end{tabular}


El modelo de regresión para el año 2010 en la ciudad de Neiva explicó el 26,05\% de los casos de dengue por la temperatura con una adecuada significancia estadística.

Se encontró que para la semana 0 por $1^{\circ} \mathrm{C}$ de temperatura media ambiental se aumentaron en 11,78 casos de dengue. Para las temperaturas de la semana 1 el modelo explicó que por $1{ }^{\circ} \mathrm{C}$ de temperatura media ambiental se aumentaron en 5 casos de dengue.

\section{ESTUDIO EXPERIMENTAL}

La observación en esta fase se realizó desde el 24 de noviembre de 2009 hasta el 29 noviembre de 2010 donde se presentaron reintentos debido al no crecimiento de los huevos a $26^{\circ} \mathrm{C}$, lo que requirió el uso de otra cohorte de huevos de cepas Rockefeller y ensayos control a temperatura ambiente para evaluar su viabilidad, evidenciándose que a condiciones ambientales el crecimiento fue normal.

En las dos generaciones observadas por cada exposición, se encontró una reducción en los tiempos de eclosión, pupa y emergimiento a $28^{\circ} \mathrm{C}$, sugiriendo estas como condiciones ideales. A $26^{\circ} \mathrm{C}$ se realizaron 2 intentos debido al no desarrollo de los huevos. A $32^{\circ} \mathrm{C}$ al parecer son condiciones adversas para el adecuado desarrollo del Aedes aegypti con una baja eclosión y alta mortalidad de los ejemplares.

Tabla 5. Modelo de regresión de incidencia de dengue y la temperatura media ambiental. Neiva Huila. Año 2010.

\begin{tabular}{|c|c|c|c|c|c|c|c|c|}
\hline Source 1 & \multicolumn{2}{|c|}{ SS } & df & MS & & \multicolumn{2}{|c|}{$\begin{array}{l}\text { Number of obs } \\
\mathrm{F}(4,47)=\end{array}$} & $\begin{array}{r}52 \\
4,14\end{array}$ \\
\hline Model & \multicolumn{2}{|c|}{20257.1634} & 4 & 5064.29084 & & \multicolumn{2}{|c|}{ Prob $>F=$} & 0,0059 \\
\hline Residual & \multicolumn{2}{|c|}{57518.7597} & 47 & 1223.8034 & & \multicolumn{2}{|c|}{ R-squared } & 0,2605 \\
\hline & & & & & & \multicolumn{2}{|c|}{ Adj R-squared } & 0,1975 \\
\hline Total & \multicolumn{2}{|c|}{77775.9231} & 51 & 1525.0181 & & \multicolumn{2}{|c|}{ Root MSE } & 34.983 \\
\hline casos 1 & \multicolumn{3}{|c|}{ Coef. } & Std. Err. & $\mathrm{t}$ & $P>1 \mathrm{t} l$ & \multicolumn{2}{|c|}{ [95\% Conf. Interval] } \\
\hline Temperatura & \multicolumn{3}{|c|}{-3.30335} & 4.943452 & $-0,67$ & 0,507 & -13.24829 & 6.641592 \\
\hline Temperatura & \multicolumn{3}{|c|}{-3.520905} & 5.530036 & $-0,64$ & 0,527 & -14.6459 & 7.604092 \\
\hline Temperatura & \multicolumn{3}{|c|}{5.007465} & 5.485055 & 0,91 & 0,366 & -6.027042 & 16.04197 \\
\hline Temperatura & \multicolumn{3}{|c|}{11.78108} & 4.776808 & 2,47 & 0,017 & 2.171378 & 21.39077 \\
\hline cons & \multicolumn{3}{|c|}{-204.1755} & 97.42013 & $-2,10$ & 0,042 & -400.1596 & -8.191503 \\
\hline
\end{tabular}

Tabla 6. Tiempo promedio del ciclo vital del Aedes aegypti en condiciones experimentales a $26^{\circ} \mathrm{C}, 28^{\circ} \mathrm{C}$ y $32^{\circ} \mathrm{C}$

\begin{tabular}{lccc}
\hline Etapa & $\mathbf{2 6 ^ { \circ } \mathrm { C }}$ & $\mathbf{2 8 ^ { \circ } \mathrm { C }}$ & $\mathbf{3 2 ^ { \circ } \mathrm { C }}$ \\
\hline Eclosión (Larva) & 4 días & 2 días & 2 días \\
Pupa & 10 días & 7 días & 18 días \\
Emergimiento (mosquito) & 4 días & 1 días & 1 días \\
\hline
\end{tabular}


El 54\% del total de ejemplares obtenidos en las diferentes exposiciones fueron hembras.

A $28^{\circ} \mathrm{C}$ se obtuvo el $54,94 \%$ del total de ejemplares, a $26^{\circ} \mathrm{C}$ el $42,92 \%$ y a $32^{\circ} \mathrm{C}$ el $5 \%$, es de anotar que a $26^{\circ} \mathrm{C}$ se necesitaron varios intentos, al igual que a $32^{\circ} \mathrm{C}$. En este último la tasa de mortalidad fue muy alta.

Al calcular el odds ratio, se encontró que la oportunidad de ser hembra Vs. macho a $26^{\circ} \mathrm{C}$ fue de 0,78 veces, la oportunidad de ser hembra Vs. macho a $28^{\circ} \mathrm{C}$ fue de 1,78 veces, es decir que tienen el $78 \%$ de posibilidad de ser hembra los huevos expuestos a $28^{\circ} \mathrm{C}$ y la oportunidad de a $32^{\circ} \mathrm{C}$ fue de 0,66 veces. (Tabla 8 ).

A $32^{\circ} \mathrm{C}$ al presentarse una tasa de mortalidad tan alta hizo que la muestra no fuera significativa.

\section{DISCUSIÓN}

La temperatura media ambiental promedio, para el año 2010 presentó un aumento de $1,12^{\circ} \mathrm{C}$ con respecto al año 2008 de acuerdo a los registros del IDEAM en la Estación Benito Salas de la ciudad de Neiva Huila Colombia. La temperatura promedio ambiental de las 24 horas del día, para el año 2010 fue de $28.10^{\circ} \mathrm{C}$, por encima del promedio de los dos años anteriores. Para los años con comportamiento típico en el canal endémico para dengue como el año 2008, el modelo de correlación calculado no explicó la incidencia de dengue por el aumento de la temperatura, debido a que no fue estadísticamente significativo. El modelo de regresión para el año 2009, en el cual se presentaron períodos epidémicos, fue estadísticamente

Tabla 7. Análisis bivariado sexo temperatura media ambiental etapa experimental.

\begin{tabular}{lcccc}
\hline & \multicolumn{3}{c}{ Temperatura } \\
\cline { 2 - 5 } género & $\mathbf{2 6 ^ { \mathbf { 0 } } \mathbf { C }}$ & $\mathbf{2 8}^{\mathbf{0}} \mathbf{C}$ & $\mathbf{3 2}^{\mathbf{0}} \mathbf{C}$ & Total \\
\hline Macho & 56 & 46 & 3 & 105 \\
Hembra & 44 & 82 & 2 & 128 \\
Total & 100 & 128 & 5 & 233 \\
\hline
\end{tabular}

Tabla 8. Cálculo de odds ratio etapa experimental.

\begin{tabular}{|c|c|c|c|c|c|c|}
\hline \multirow{2}{*}{$\frac{\text { Tempera a }}{26^{\circ} \mathrm{C}}$} & \multirow{2}{*}{$\begin{array}{c}\text { cases } \\
44\end{array}$} & \multicolumn{2}{|c|}{ controls } & \multirow{2}{*}{$\begin{array}{c}\text { odds } \\
0,78571\end{array}$} & \multicolumn{2}{|c|}{$[95 \%$ Conf. Interval $]$} \\
\hline & & & 56 & & 0,52940 & 1,16612 \\
\hline $26^{\circ} \mathrm{C}$ & 82 & & 46 & 1,78261 & 1,24238 & 2,55775 \\
\hline $32^{\circ} \mathrm{C}$ & 2 & & 3 & 0,66667 & 0,11140 & 3,98975 \\
\hline Test of homogeneity (equa & ds): & $\begin{array}{l}\operatorname{chi} 2(2) \\
\text { Pr }>\text { chi2 }\end{array}$ & ) & $\begin{array}{r}9,55 \\
0,0085\end{array}$ & & \\
\hline Score test for trend of odds: & & $\begin{array}{l}\operatorname{chi} 2(1) \\
\operatorname{Pr}>\text { chi2 }\end{array}$ & $\begin{array}{l}\text { ) } \\
2=\end{array}$ & $\begin{array}{r}6,30 \\
0,0121\end{array}$ & & \\
\hline
\end{tabular}


significativo, explicando el 33,10\% del comportamiento de la incidencia de dengue por las temperaturas, principalmente las condiciones de temperatura media ambiental de dos semanas antes de la presentación de los casos, durante cuyo período, según el modelo, el aumento de un grado centígrado $\left(1^{\circ} \mathrm{C}\right)$ de temperatura explicó el aumentó 3,5 casos de dengue con una p de 0,040. El modelo de regresión para el año 2010 en la ciudad de Neiva explicó el 26,05\% de los casos de dengue por el aumento de la temperatura, con una adecuada significancia estadística. Se encontró que para la semana 0 por $1^{\circ} \mathrm{C}$ de temperatura media ambiental se aumentaron en 11,78 casos de dengue. Para las temperaturas de la semana 1 por $1^{\circ} \mathrm{C}$ de temperatura media ambiental se aumentaron en 5 casos de dengue. No se encontraron bases de datos confiables anteriores al inicio de operación del software SIVIGILA a partir del año 2008. A partir de este año, se tomó esta base por tener mayores validaciones en su proceso. Se consideró la humedad relativa ambiental como constante ya que ésta depende de la temperatura media ambiental, sin embargo esto podría generar un potencial sesgo de medición. En el estudio experimental esta variable fue controlada externamente. Se presentaron dificultades durante el estudio experimental por la no eclosión de los huevos en un primer ensayo a $26^{\circ} \mathrm{C}$ y sobre todo a $32^{\circ} \mathrm{C}$, lo que prolongó los tiempos de observación de las dos generaciones expuestas a cada rango de temperatura. Se encontró un aparente favorecimiento de condiciones a $28^{\circ} \mathrm{C}$ debido a una reducción significativa en los tiempos de eclosión, pupa y emergimiento de los huevos expuestos. La exposición a $26^{\circ} \mathrm{C}$ hizo más difícil el desarrollo de los huevos y a $32^{\circ} \mathrm{C}$ sugiere que son condiciones adversas para su desarrollo debido a la baja eclosión, emergimiento y alta mortalidad. En el estudio experimental se encontró que la oportunidad de ser hembra Vs macho a $28^{\circ} \mathrm{C}$ es $78 \%$, cuyo valor es superior en comparación a los obtenidos a $26^{\circ} \mathrm{C}$ y $32^{\circ} \mathrm{C}$ de exposición. A $32^{\circ} \mathrm{C}$ no fue posible el cálculo por cuanto la mortalidad de los ejemplares hizo insuficiente la muestra. Los resultados obtenidos en condiciones experimentales controlan variables que en estado natural afectan los resultados en el desarrollo del Aedes aegypti sin embargo el cálculo de determinación y modelo de correlación encontrada en la primera etapa para los años 2009 y 2010 con comportamiento epidémico atípico, así como el comportamiento de las variables climáticas en ese mismo período de tiempo, sugieren una posible relación entre la temperatura media ambiental y la incidencia del dengue. Bajo condiciones experimentales se encontró cierto favorecimiento 4:3 de desarrollo de hembras Vs. machos a $30^{\circ} \mathrm{C}$ de exposición ${ }^{(28)}$, que podrían correlacionarse con los resultados obtenidos a $28^{\circ} \mathrm{C}$ en el estudio experimental.

\section{CONCLUSIONES}

El presente estudio plantea limitaciones por enfocar la relación hacia la variable temperatura media ambiental al considerarla una variable independiente; ya que el comportamiento de las epidemias de dengue pueden afectarse por múltiples variables, lo cual limita los resultados de la investigación

De acuerdo a los resultados encontrados se concluye que quizá podría existir una correlación entre el aumento de la temperatura media ambiental crítica sostenida de $28^{\circ} \mathrm{C}$ y el comportamiento de las epidemias de dengue, estos datos fueron coincidentes con los hallazgos de exposición a $28^{\circ} \mathrm{C}$ encontrados en la etapa experimental, lo que puede otorgarle una condición de variable predictiva de epidemias de dengue a la temperatura media ambiental. La temperatura media ambiental de $28^{\circ} \mathrm{C}$ en condiciones experimentales favorece el desarrollo de hembras Vs. machos en comparación a $26^{\circ} \mathrm{C}$ y $30^{\circ} \mathrm{C}$ concordante con hallazgos de estudios experimentales ${ }^{(29)}$. Con lo anterior se propone que ante el aumento esperado de la temperatura media ambiental producto del efecto invernadero 
para las próximas décadas, se podría esperar una afectación sustancial de los patrones endémicos epidémicos de las enfermedades tropicales transmitidas por vectores y en este caso las relacionadas con el Aedes aegypti ya que al facilitar el ciclo de vida del vector y con un aumento en la población de hembras, la probabilidad de transmisión del virus del dengue facilitará la presentación de períodos epidémicos atípicos como el presentado en el segundo semestre del 2009 y el primer semestre de 2010. La temperatura ideal para el Aedes aegypti en condiciones experimentales es de $28^{\circ} \mathrm{C}$ con reducción en el tiempo del ciclo vital. Si la temperatura aumenta a promedio superiores a $30^{\circ} \mathrm{C}$ quizá puede alterar negativamente el desarrollo de las especies de vectores que parecen tener mayor susceptibilidad y lenta adaptación a temperaturas tan altas, con la consecuente alteración de los microecosistemas. La temperatura media ambiental puede jugar un importante papel en la definición del sexo en el Aedes aegypti como se ha evidenciado en investigaciones en algunas especies de tortugas y lagartos, lo que motiva el desarrollo de más estudios para aclarar esta asociación(30-34).

\section{AgRADECIMIENTOS}

A la Secretaría de Salud del Departamento del Huila Colombia quienes facilitaron su infraestructura, información y personal para el desarrollo del presente proyecto. A la Dra. Evelina Chapman y al Dr. Sergio Muñoz por su asesoría científica durante la presente investigación.

\section{REFERENCIAS}

1. Cambio climático y biodiversidad. Grupo intergubernamental de expertos sobre el Cambio Climático. Organización de las Naciones Unidas. Disponible en http://www.ipcc.ch/ pdf/technical-papers/climate-changesbiodiversity-sp.pdf

2. Beserra EB, de Castro FP Jr, dos Santos JW, Santos Tda S, Fernandes CR. [Biology and thermal exigency of Aedes aegypti (L.) (Diptera: Culicidae) from four bioclimatic localities of Paraíba]. Neotrop Entomol 2006 Nov-Dec;35(6):853-860.

3. Monteiro LC, de Souza JR, de Albuquerque CM. Eclosion rate, development and survivorship of Aedes albopictus (Skuse)(Diptera: Culicidae) under different water temperatures. Neotrop Entomol 2007 Nov-Dec;36(6):966-971.

4. Mourya DT, Yadav P, Mishra AC. National Institute of Virology, Pune, India. Effect of temperature stress on immature stages and susceptibility of Aedes aegypti mosquitoes to chikungunya virus. Am J Trop Med Hyg 2004 Apr;70(4):346-350.

5. Teng HJ, Apperson CS. Department of Entomology, North Carolina State University, Raleigh 27695-7647, USA Development and survival of immature Aedes albopictus and Aedes triseriatus (Diptera: Culicidae) in the laboratory: effects of density, food, and competition on response to temperature. J Med Entomol 2000 Jan;37(1):40-52.

6. Watts DM, Burke DS, Harrison BA, Whitmire RE, Nisalak A Am J Effect of temperature on the vector efficiency of Aedes aegypti for dengue 2 virus. Trop Med Hyg 1987 Jan;36(1):143-152.

7. Alto BW, Juliano SA. J Temperature effects on the dynamics of Aedes albopictus (Diptera: Culicidae) populations in the laboratory. Med Entomol 2001 Jul;38(4):548-556.

8. Tun-Lin W, Burkot TR, Kay BH. Effects of temperature and larval diet on development rates and survival of the dengue vector Aedes aegypti in north Queensland, Australia Effects of temperature and larval diet on development rates and Survival of the dengue vector Aedes aegypti in north Queensland, Australia Med Vet Entomol 2000 Mar;14(1):31-37.

9. Fernanda Silva Costa; Juliana Junqueira da Silva; Carina Mara de Souza; Júlio Mendes Rev. Soc. Bras Dinâmica populacional de Aedes aegypti (L) em área urbana de alta incidência de dengue. Med Trop vol.41 no.3 Uberaba May/ June 2008.

10. US Naval Medical Research Unit No 2 Climatic factors associated with epidemic dengue in Palembang, Indonesia: implications of short-term meteorological events on virus transmission. 
Southeast Asian J Trop Med Public Health 2006 Nov;37(6):1103-1116.

11. Rifakis Pedro, Gonçalves Nacary, Omaña Wilberto, Manso Miguel, Espidel Alberto, Intingaro Alfonso Asociación entre las variaciones climáticas y los casos de dengue en un hospital de Caracas, Venezuela, 1998-2004. Rev Peru Med Exp Salud Pública 2005;22(3).

12. Michael J Bangs, Ria P Larasati1, Andrew L Corwin. Suharyono Wuryadi Climatic factors associated with epidemic dengue in Palembang, Indonesia: implications of short-term meteorological events on virus transmission. Southeast Asian J Trop Med Public Health 2006 Nov; 37(6):1103-1116.

13. Kovats RS. Department of Epidemiology and Population Health El Niño and human health. London School of Hygiene and Tropical Medicine, England. Bull World Health Organ. 2000;78(9):1127-1135.

14. Hurtado-Díaz M, Riojas-Rodríguez $H$, Rothenberg SJ, Gomez-Dantés H, Cifuentes E.Short communication: impact of climate variability on the incidence of dengue in Mexico. Trop Med Int Health 2007 Nov;12(11):1327-37. Epub 2007 Oct 23.

15. Ribeiro AF, Marques GR, Voltolini JC, Condino ML. Association between dengue incidence and climatic factors Rev Saude Publica 2006;40(4):671-676.

16. Rico Johansson MA, Dominici F, Glass GE PLoS. Local and global effects of climate on dengue transmission in puerto Negl Trop Dis 2009;3(2): e382.

17. Hales S, Weinstein P; Souares Y, Woodward A Environ Health Perspect, El Niño and the dynamics of vectorborne disease transmission Environ Health Perspect 1999 Feb;107(2):99-102.

18. JS, Prevots DR, Vaca Marin MA, Gomez Dantes $\mathrm{H}$, Zarate Aquino ML, Longini IM Jr, Sepulveda Amor J. Determinants and predictors of dengue infection in Mexico.Koopman Department of Epidemiology, University of Michigan, Ann Arbor 48109-2029. Am J Epidemiol 1991 Jun 1;133(11):1168-1178.

19. Gubler DJ. Aedes aegypti and Aedes aegyptiborne disease control in the 1990s: top down or bottom up. Am J Trop Med Hyg 1989;40(6): 571-578.

20. Isberg SR Johnston SM, Chen Y Moran C. Centre for Advanced Technologies in Animal Genetics and Reproduction, Faculty of Veterinary Science, University of Sydney, First evidence of higher female recombination in a species with temperature-dependent sex determination: the saltwater crocodile NSW, Australia Journal of Heredity 2006 97(6):599-602. Publicado online el 17 de noviembre de 2006.

21. Crews D.Section of Integrative Biology, University of Texas at Austin, Austin, TX 78712, USA.Sex determination: where environment and genetics meet. Evol Dev 2003 Jan-Feb;5(1):50-55.

22. C. M. Johnston, M. Barnett, P. T. Sharpe, J. A. M. Graves, M. B. Renfree, B. Capel, D The Molecular Biology of Temperature-Dependent Sex Determination [and Discussion]. Mireille Philosophical Transactions: Biological Sciences, Vol. 350, No. 1333, Mechanisms in Vertebrate Sex Determination Nov. 29, 1995;297-304.

23. Western PS, Harry JL, Graves JA, Sinclair AH. Department of Paediatrics, University of Melbourne, Royal Children's Hospital, Victoria, Australia Temperature-dependent sex determination in the American Alligator: AMH precedes SOX9 expression. Dev Dyn 1999 Dec;216(4-5):411-419.

24. Instituto Nacional de Salud de Colombia. Procedimiento Manejo de colonias de insectos en el laboratorio de entomología. Código: INT R01-003-5010-003.

25. Asociación Médica Mundial. Declaración de Helsinki.

26. Organización de las Naciones Unidas. Declaración Universal de los derechos del animal.

27. Conferencia Mundial sobre la Ciencia para el Siglo XXI: Un nuevo compromiso Declaración de Budapest. Declaración sobre la Ciencia y el uso del saber científico.

28. Tun-Lin W, Burkot TR, Kay BH. Effects of temperature and larval diet on development rates and survival of the dengue vector Aedes aegypti in north Queensland, Australia. Med Vet Entomol 2000 Mar;14(1):31-37.

29. US Naval Medical Research Unit No 2 Climatic factors associated with epidemic dengue in Palembang, Indonesia: implications of short-term meteorological events on virus transmission. Southeast Asian J Trop Med Public Health 2006 Nov; 37(6):1103-1116.

30. Mourya DT, Yadav P, Mishra AC. National Institute of Virology, Pune, India. Effect of temperature stress on immature stages and susceptibility of Aedes aegypti mosquitoes to chikungunya virus. Am J Trop Med Hyg 2004 Apr; 70(4):346-350. 
31. Teng HJ, Apperson CS. Department of Entomology, North Carolina State University, Raleigh 27695-7647, USA Development and survival of immature Aedes albopictus and Aedes triseriatus (Diptera: Culicidae) in the laboratory: effects of density, food, and competition on response to temperature. J Med Entomol. 2000 Jan; 37(1):40-52.

32. Monteiro LC, de Souza JR, de Albuquerque CM. Graduanda do Curso de Ciências Biológicas, Univ Federal de Pernambuco, Recife, PE, Brazil Eclosion rate, development and survivorship of
Aedes albopictus (Skuse)(Diptera: Culicidae) under different water temperatures. Neotrop Entomol 2007 Nov-Dec; 36(6):966-971.

33. Watts DM, Burke DS, Harrison BA, Whitmire RE, Nisalak A Am J Effect of temperature on the vector efficiency of Aedes aegypti for dengue 2 virus. Trop Med Hyg 1987 Jan;36(1):143-152.

34. Alto BW, Juliano SA. J Temperature effects on the dynamics of Aedes albopictus (Diptera: Culicidae) populations in the laboratory. Med Entomol $2001 \mathrm{Jul} ; 38(4): 548-556$. 